\title{
Manejo nutricional em pacientes com risco de síndrome de realimentação
}

\author{
Nutritional management in patients at risk of refeeding syndrome
}

\section{DOI: $10.37111 /$ braspenj.AE2019344001}

\section{Matheus Horta Sad'}

Bruna Fernanda Camargo Silva Parra²

Ricardo Ferrer ${ }^{3}$

Antônio Valério da Silva Júnior ${ }^{4}$

Flávia Julie do Amaral Pfeilsticker ${ }^{5}$

Liane Brescovici Nunes de Matos ${ }^{6}$

Diogo Oliveira Toledo’

\section{Unitermos:}

Síndrome da realimentação. Terapia nutricional. Transtornos nutricionais

\section{Keywords:}

Refeeding syndrome. Nutrition therapy. Nutrition disorders

\section{Endereço para correspondência:}

Diogo Oliveira Toledo

Rua Dr. Alceu de Campos Rodrigues, 95 - Vila Nova

Conceição - São Paulo, SP, Brasil - CEP: 04544-000

E-mail:diogootoledo@gmail.com

\section{Submissão}

27 de agosto de 2019

Aceito para publicação

12 de dezembro de 2019

\section{RESUMO}

A síndrome de realimentação (SR) é uma complicação ameaçadora à vida, que ocorre após jejum prolongado em pacientes desnutridos ou após processos catabólicos graves. Geralmente ocorre nas primeiras 72 horas após o início da dieta enteral ou parenteral e acomete até um terço dos pacientes. Os fatores de risco importantes são índice de massa corporal (IMC) baixo, perda de peso não intencional, baixo ou nenhum aporte calórico por pelo menos 5 dias, história de abuso de álcool ou drogas e distúrbios eletrolíticos de base. É obrigatória a prescrição de tiamina intravenosa e a reposição de eletrólitos (fósforo, potássio e magnésio). O início e a progressão da terapia nutricional deve ser gradual e a quantidade de calorias ofertada deve ser pequena nos primeiros dias com monitorização diária de eletrólitos. Caso ocorra queda nos valores de eletrólitos, especialmente hipofosfatemia, sugere-se reduzir a infusão de dieta e progredir de forma lenta ao longo de 48 horas. A SR merece atenção especial da equipe de terapia nutricional, com objetivo de prevenção, identificação e tratamento precoce.

\section{ABSTRACT}

Refeeding syndrome (RS) is a life-threatening complication that occurs after prolonged starvation in malnourished patients or after severe catabolic events. It usually happens in the first 72 hours after the beginning of either enteral or parenteral nutrition and can affect one third of the patients. The most important risk factors are low body mass index (BMI), unintentional weight loss, none or low caloric supply for at least 5 days, history of alcohol or drug abuse and baseline electrolyte disturbances. Thiamine and electrolyte (phosphate, potassium and magnesium) supplementation is mandatory. The initiation and progression of nutrition therapy must be gradual with low amount of calories in the first days, with daily electrolyte monitoring. In the case of low electrolyte levels, especially hypophosphatemia, diet infusion should be reduced and slowly increased over 48 hours. RS deserves special attention the nutrition team to be prevented, identified and treated early.

1. Médico Intensivista pela AMIB. Especialista em Nutrição Parenteral e Enteral pela BRASPEN/SBNPE, São Paulo, SP, Brasil.

2. Especialista em Nutrição parenteral e enteral pela BRASPEN/SBNPE. Pós-graduada em Terapia Nutricional em Cuidados Intensivos pelo GANEP, São Paulo, SP, Brasil.

3. Graduação em Enfermagem pelo Centro Universitário São Camilo, São Paulo, SP. Especialização em Nutrição Clínica e Hospitalar - GANEP Nutrição Humana. Especialista em Nutrição Parenteral e Enteral pela BRASPEN/SBNPE.

4. Médico intensivista pela Irmandade Santa Casa de Misericórdia de São Paulo. Pós-graduação latu sensu em Nutrologia pela ABRAN, São Paulo, SP, Brasil.

5. Médica intensivista pela AMIB. Pós graduação em Nutrologia para o paciente grave pelo Hospital Israelita Albert Einstein. Especialista em Nutrição enteral e parenteral pela BRASPEN/ SBNPE, São Paulo, SP, Brasil.

6. Médica Intensivista pela AMIB. Especialista em Nutrição Parenteral e Enteral pela BRASPEN/SBNPE e ABRAN, São Paulo, SP, Brasil.

7. Médico Intensivista pela AMIB. Nutrólogo pela ABRAN, São Paulo, SP, Brasil. 


\section{INTRODUÇ̃̃O}

A síndrome de realimentação (SR) é uma reação anabólica causada pela terapia nutricional' ${ }^{1}$. É uma complicação potencialmente ameaçadora à vida, que ocorre após jejum prolongado em pacientes desnutridos ou após processos catabólicos graves ${ }^{1-3}$.

A incidência da SR varia de $14 \%$ a $28 \%$ e ocorre após o início da dieta (seja enteral ou parenteral), sendo importante identificar grupos de risco².

Clinicamente, a SR pode apresentar um amplo espectro, incluindo desde formas oligosintomáticas até quadros graves, com deterioração clínica e morte cardíaca súbita. Em geral, uma maior mortalidade é encontrada em populações específicas, particularmente idosos, portadores de HIV e doentes críticos'.

Embora a terapia nutricional seja um dos tratamentos hospitalares mais usados no mundo, a SR ainda é pouco estudada na população internada'.

\section{FISIOPATOLOGIA}

A fisiopatologia da SR não é completamente conhecida, mas geralmente ocorre 72 horas após o início da terapia nutricional ${ }^{1-3}$.

Durante o período de jejum, a oxidação da glicose é reduzida. A secreção de insulina é, portanto, diminuída e os níveis de glucagon e catecolaminas aumentam, depletando as reservas de glicogênio. A gliconeogênese começa junto com a lipólise e a proteólise, para manter a produção de energia. Como resultado, as proteínas musculares são consumidas, bem como as vitaminas e eletrólitos. Através da lipólise, os níveis de ácidos graxos livres aumentam e a cetogênese no fígado é estimulada. Portanto, corpos cetônicos, principalmente hidroxibutirato, tornam-se os principais fornecedores de energia do organismo'.

Com o início da dieta, a concentração de glicose repentinamente aumenta e a secreção de insulina estimula os processos anabólicos. Ocorre, portanto, fluxo intracelular de glicose e eletrólitos (fósforo, potássio, magnésio), levando à queda nos seus níveis séricos, o que pode precipitar complicações. Além disso, há uma tendência à retenção de sódio e água'.

A queda dos níveis de eletrólitos e vitaminas geralmente surge, principalmente, devido à falta de tiamina. A tiamina é um importante co-fator no metabolismo dos carboidratos, permitindo a conversão de glicose em ATP (ciclo de Krebs). Nos casos de deficiência de tiamina, a glicose é convertida em lactato pela lactato desidrogenase, levando à acidose metabólica' .

\section{QUADRO CLÍNICO E DIAGNÓSTICO}

A nomenclatura "SR iminente" é usada quando o quadro se restringe à presença dos distúrbios eletrolíticos (hipofosfatemia, hipocalemia e/ou hipomagnesemia). Se, associado a eles surgem sintomas clínicos, o quadro leva o nome de "SR manifesta"2.

Não existe um exame padrão-ouro para o diagnóstico de SR, sendo, portanto, necessário alto grau de suspeição.

As manifestações clínicas mais comuns são inespecíficas e incluem edema, taquicardia e taquipneia ${ }^{1-4}$.

A deficiência de tiamina pode levar a alterações neurológicas (encefalopatia de Wernicke, beribéri seco) ou distúrbios cardiovasculares (beribéri úmido)'.

Hipofosfatemia grave pode levar a comprometimento da função neuromuscular, com parestesias, confusão mental, convulsões, coma, cãibras e fraqueza muscular. No sistema respiratório, causa hipoventilação e, eventualmente, insuficiência respiratória aguda. A rabdomiólise também foi descrita como resultado de hipofosfatemia grave ${ }^{1}$. A deficiência de fósforo também pode causar trombocitopenia, coagulopatia, disfunção leucocitária e eritrocítica?.

Hipomagnesemia e hipocalemia podem levar a arritmias cardíacas graves e alterações neuromusculares, como fraqueza, paralisia, parestesia, confusão, rabdomiólise e depressão respiratória. Na desnutrição grave e prolongada, pode haver atrofia cardíaca, com bradicardia sinusal e aumento do intervalo QTc. Essas mudanças tornam o coração mais vulnerável à hipofosfatemia e a hipocalemia, com risco de arritmias ventriculares e morte súbita?.

\section{FATORES DE RISCO}

A estratificação dos pacientes em grupos de risco auxilia no manejo da terapia nutricional2 ${ }^{2}$ (Figura 1).

Os fatores mais relevantes são índice de massa corporal (IMC) baixo $(<18,5)$, perda de peso não intencional (pelo menos 10\% nos últimos 3-6 meses), baixo ou nenhum aporte calórico por pelo menos 5 dias, história de abuso de álcool ou drogas e distúrbios eletrolíticos de base ${ }^{5}$.

\section{PREVENÇÃO E TRATAMENTO}

Em todo paciente com risco de SR, antes do início da dieta, é necessária a prescrição de tiamina intravenosa (100-300 mg/dia) e dosagem de eletrólitos, com especial atenção ao fósforo ${ }^{1-6}$.

A reposição de eletrólitos é sugerida mesmo se o nível sérico estiver normal ou no limite inferior da normalidade, devido ao alto risco de queda ${ }^{1,2}$.

A quantidade de calorias ofertada aos pacientes em risco deve ser pequena nos primeiros dias e de progressão gradual, habitualmente levando-se mais tempo (5-10 dias) para se atingir a meta (Figura 2$)^{2}$. 


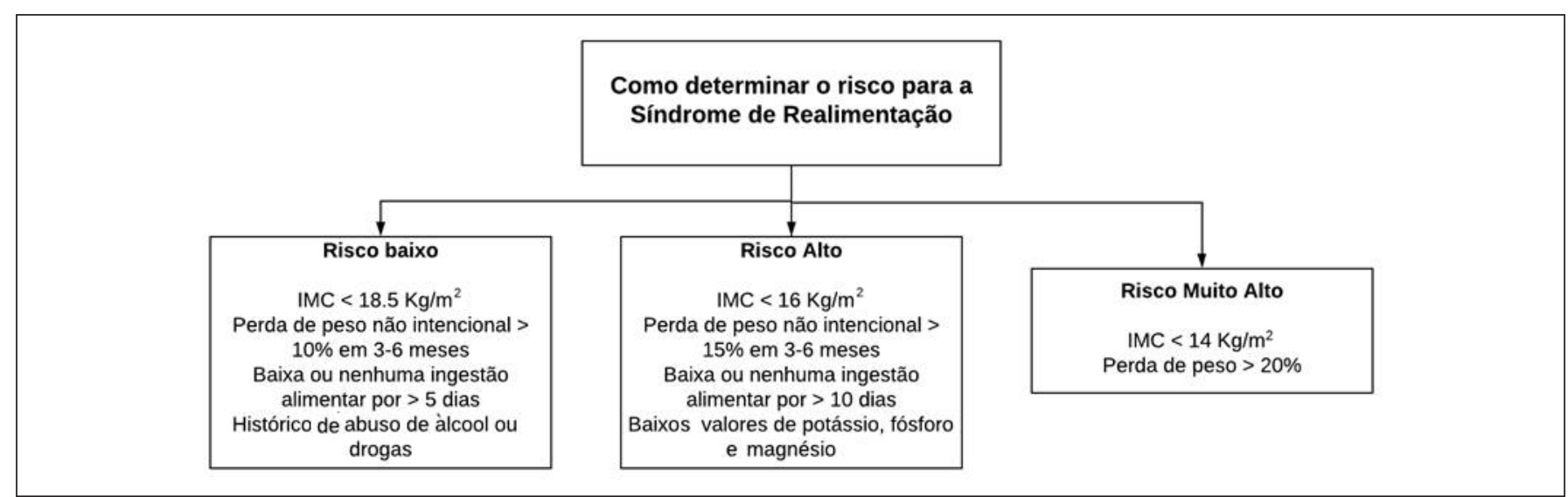

Figura 1 - Determinar o risco para SR. IMC = indice de massa corporal. Adaptado de Aubry et al. ${ }^{2}$.

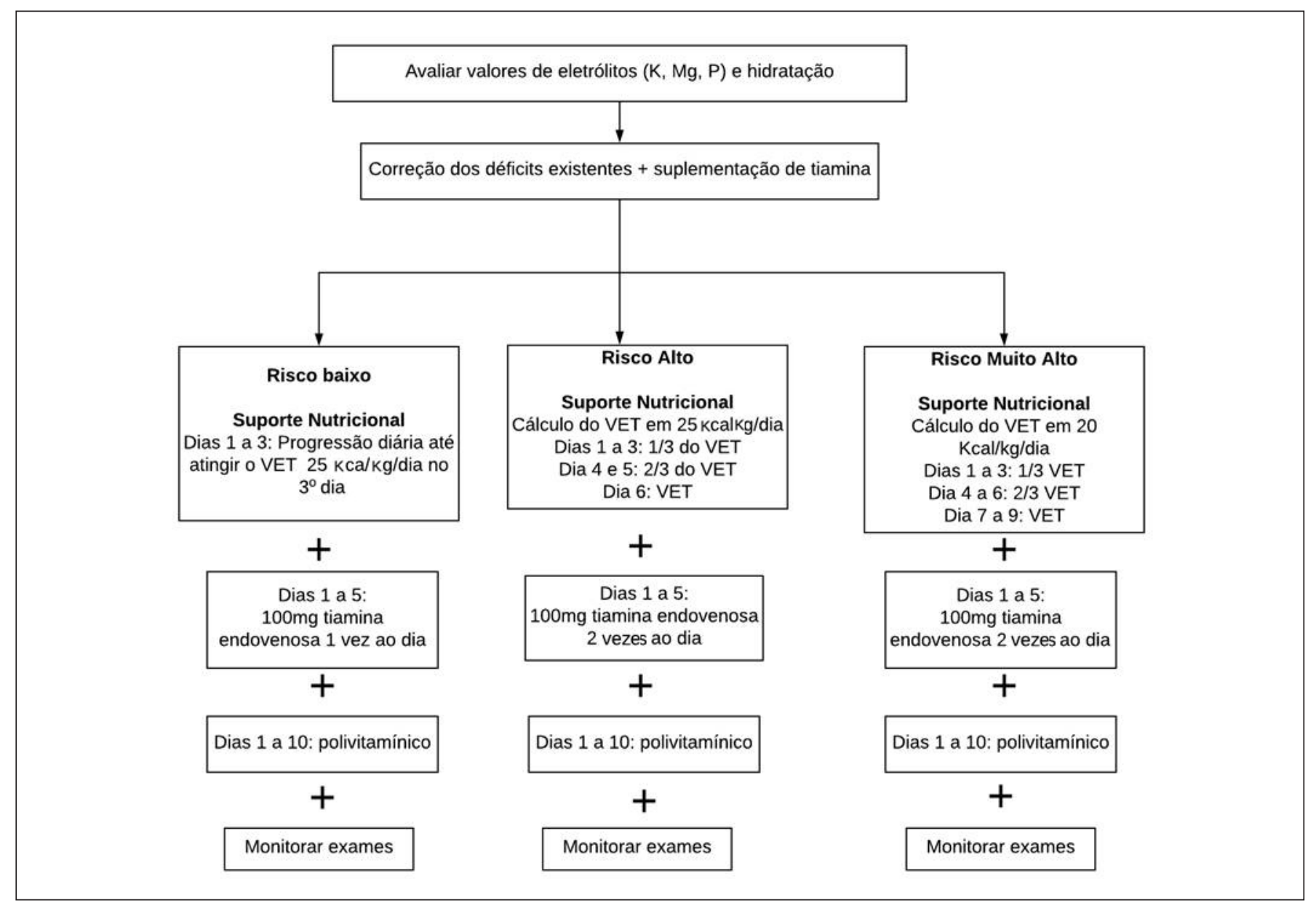

Figura 2 - Manejo da terapia nutricional nos pacientes com risco de SR. VET = valor energético total. Adaptado de Aubry et al. ${ }^{2}$

Nesse contexto, é fundamental a monitorização diária de eletrólitos, sinais vitais e peso, especialmente nas primeiras 72 horas $^{2}$.

Caso haja redução no nível sérico de eletrólitos após o início da dieta, é recomendada a redução do aporte calórico. Em um estudo randomizado com 339 pacientes críticos que apresentaram hipofosfatemia após a terapia nutricional, foi observada maior sobrevida em 60 dias no grupo que reduziu a oferta da dieta para $500 \mathrm{kcal}$ por dia por 48 horas $^{3}$.

\section{CONCLUSÃO}

A SR é uma entidade relativamente frequente e potencialmente fatal. Sendo assim, deve ser dada atenção especial para a sua prevenção, identificação e tratamento precoces. 
Pacientes em inanição prolongada ou sob estados catabólicos prolongados devem ser considerados sob risco. Esses pacientes se beneficiam de menor aporte calórico, com aumento gradual da terapia nutricional, sob estrita monitorização de eletrólitos e suplementação de tiamina e polivitamínico.

\section{REFERÊNCIAS}

1. Friedli N, Stanga Z, Culkin A, Crook M, Laviano A, Sobotka $\mathrm{L}$, et al. Management and prevention of refeeding syndrome in medical inpatients: an evidence-based and consensus-supported algorithm. Nutrition. 2018;47:13-20.
2. Aubry E, Friedli N, Schuetz P, Stanga Z. Refeeding syndrome in the frail elderly population: prevention, diagnosis and management. Clin Exp Gastroenterol. 2018;11:255-64.

3. Doig GS, Simpson F, Heigles PT, Bellomo R, Chesher D, Caterson ID, et al. Restricted versus continued standard caloric intake during the management of refeeding syndrome in critically ill adults: a randomised, parallel-group, multicentre, single-blind controlled trial. Lancet Respir Med. 2015;3(12):943-52.

4. Koekkoek WAC, Van Zanten ARH. Is refeeding syndrome relevant for critically ill patients? Curr Opin Clin Nutr Metab Care. 2018;21(2):130-7.

5. National Collaborating Centre for Acute Care. Nutrition support for adults: oral nutrition support, enteral tube feeding and parenteral nutrition. London: National Collaborating Centre for Acute Care; 2006.

6. Berger MM, Reintam-Blaser A, Calder PC, Casaer M, Hiesmayr MJ, Mayer K, et al. Monitoring nutrition in the ICU. Clin Nutr. 2019;38(2):584-93.

Local de realização do estudo: Departamento de Terapia Nutricional do Hospital São Luiz, São Paulo, SP, Brasil.

Conflito de interesse: Os autores declaram não haver. 